International Journal of Advanced Research in Engineering and Technology (IJARET) Volume 10, Issue 3, May-June 2019, pp. 103-111, Article ID: IJARET_10_03_011

Available online at http: //iaeme. com/Home/issue/IJARET?Volume=10\&Issue=3

ISSN Print: 0976-6480 and ISSN Online: 0976-6499

(C) IAEME Publication

\title{
Variety of monoid recognizable $l$-fuzzy languages
}

\author{
Ajitha Kumari $\mathrm{K}^{*} \&$ Ramesh Kumar $\mathrm{P}^{\dagger}$ \\ Department of Mathematics, B.J.M.Govt.College, Chavara, Kollam, Kerala, 691583, India \\ Department of Mathematics, University of Kerala, Thiruvananthapuram, Kerala 695581, India.
}

\begin{abstract}
Here we study $l$-fuzzy languages recognized by finite monoids. We show that the class of monoid recognizable $l$-fuzzy languages is closed under scalar products, quotients, inverse homomorphic images and $c$-cuts. We introduce the notion of variety of monoid recognizable $l$-fuzzy languages. Also we obtain an Eilenberg type variety theorem for $l$-fuzzy languages.

Key words : $l$-fuzzy languages, Syntactic Congruence, Variety of $l$-fuzzy languages.

2000 Mathematics Subject Classification : 20Mxx, 68Q45, 03E72.
\end{abstract}

\section{Introduction}

Fuzzy sets were introduced by Zadeh in [7]. The theory of fuzzy language was developed as a generalization of the classical notion of (crisp)languages. The concept of fuzzy automaton was introduced by Wee in 1967. More on recent developments in the theory of automata and fuzzy languages was given in [3]. Petkovic [4] introduced the concept of regular (recognizable) fuzzy languages as generalization of regular languages. In [4], he introduced the notion of syntactic monoid of a fuzzy languages and proved that the syntactic monoid of a recognizable fuzzy language is finite. Also he proved that the class of all recognizable fuzzy languages is a variety and there is a one-one correspondence between the variety of all recognizable fuzzy languages and the pseudovariety of finite monoids. We generalized the notion of monoid recognizability and syntactic monoid etc to the class of $l$-fuzzy languages in [1].

In this paper we introduce the notion of variety of monoid recognizable $l$-fuzzy languages. We prove that there exists a mutually inverse lattice isomorphism between the lattices of all varieties of $l$-fuzzy languages and all varieties of crisp languages. Using this results and Eilenberg variety theorem we establish a one to one correspondence between the lattices of all varieties of $l$-fuzzy languages and all varieties of finite monoids.

\section{Preliminaries}

In this section we recall the basic definitions, results and notations that will be used in the sequel. All undefined terms are as in $[2,3,5,6]$. A lattice is a partially ordered set in which every subset consisting of two element has a least upper bound and a greatest lower bound. A lattice $l$ is said to be distributive if elements of $l$ satisfies distributive properties.

i) $a \wedge(b \vee c)=(a \wedge b) \vee(a \wedge c)$.

*email : ambiliragamalika@gmail.com

†email : ramesh.ker64@gmail.com 
ii) $a \vee(b \wedge c)=(a \vee b) \wedge(a \vee c)$

A lattice $l$ is said to be bounded if it has a greatest element 1 and a least element 0 . Let $l$ be a bounded lattice and let $a \in l$. An element $b \in l$ is called complement of $a$ if $a \vee b=1$ and $a \wedge b=0$. A lattice $l$ is called complemented if it is bounded and if every element in $l$ has a complement. A lattice $l$ is called a complete lattice if every nonempty subset of $l$ has greatest lower bound and least upper bound in $l$.

A nonempty set $M$ with an associative binary operation is called a semigroup. Semigroups that have an identity element are called monoids.

Let $A$ be a nonempty finite set, called an alphabet. Elements of $A$ are called letters. A finite sequence of letters of $A$ is called a word. The length of the word $w$ is the number of letters of $A$ occurring in $w$. A word of length zero is called empty word and is denoted by $\varepsilon . A^{+}$denotes the set of all nonempty words over an alphabet $A$ and $A^{*}=A^{+} \cup\{\varepsilon\}$ is a monoid under the operation concatenation, called free monoid over $A$. A subset of $A^{*}$ is called the language $L$ over an alphabet $A$.

A $*$-variety of languages is a class of recognizable languages $A^{*} \mathscr{V}$ such that

i) For every alphabet $A, A^{* \mathscr{V}}$ is a Boolean algebra.

ii) If $\varphi: A^{*} \rightarrow B^{*}$ is a homomorphism and if $L \in B^{*} \mathscr{V}$, then $L \varphi^{-1} \in A^{*} \mathscr{V}$.

iii) If $L \in A^{*} \mathscr{V}$ and $a \in A$, then both $a^{-1} L=\left\{u \in A^{*} \mid a u \in L\right\}$ and $L a^{-1}=\left\{u \in A^{*} \mid u a \in L\right\}$ are in $A^{* \mathscr{V}}$.

Let $l$ be a complete complemented distributive lattice. Any function $\lambda$ from $A^{*}$ into $l$ is called a $l$-fuzzy language over the alphabet $A$.

The complement $\bar{\lambda}$ of a $l$-fuzzy language $\lambda$ is defined as

$$
\bar{\lambda}(u)=\overline{\lambda(u)}
$$

where $\overline{\lambda(u)}$ denotes the complement of $\lambda(u)$ in $l$.

For $l$-fuzzy languages $\lambda_{1}, \lambda_{2}$ over $A$, their join $(\vee)$ and meet $(\wedge)$ are defined by

$$
\left(\lambda_{1} \vee \lambda_{2}\right)(u)=\lambda_{1}(u) \vee \lambda_{2}(u)
$$

and

$$
\left(\lambda_{1} \wedge \lambda_{2}\right)(u)=\lambda_{1}(u) \wedge \lambda_{2}(u)
$$

Definition 2.1 (cf.[1], Definition 3.2). Let $\lambda$ be a l-fuzzy language over an alphabet $A$. Then $\lambda$ is recognizable if there exist a finite monoid $M$, a homomorphism $\phi: A^{*} \rightarrow M$ and a l-fuzzy subset $\pi: M \rightarrow l$ such that $\lambda=\pi \phi^{-1}$ where $\pi \phi^{-1}(u)=\pi(\phi(u)), u \in A^{*}$.

The class of all recognizable $l$-fuzzy languages over $A$ is denoted by $l F\left(A^{*}\right)$.

Theorem 2.2 (cf. [1], Theorem 3.4). Let $\lambda, \lambda_{1}, \lambda_{2}$ be recognizable l-fuzzy languages over an alphabet $A$. Then we have the following

(i) $\lambda_{1} \vee \lambda_{2}$ is recognizable.

(ii) $\lambda_{1} \wedge \lambda_{2}$ is recognizable.

(iii) $\bar{\lambda}$ is recognizable. 
Variety of monoid recognizable $l$-fuzzy languages

\section{Quotient, Scalar product, c-cut and Inverse homomorphic image of l-fuzzy languages}

Let $\lambda_{1}, \lambda_{2}$ be $l$-fuzzy languages over $A$. Then their left and right quotients are defined by

$$
\left(\lambda_{1}^{-1} \lambda_{2}\right)(u)=\bigvee_{v \in A^{*}}\left(\lambda_{2}(v u) \wedge \lambda_{1}(v)\right), u \in A^{*}
$$

and

$$
\left(\lambda_{2} \lambda_{1}^{-1}\right)(u)=\bigvee_{v \in A^{*}}\left(\lambda_{2}(u v) \wedge \lambda_{1}(v)\right), u \in A^{*} .
$$

Let $c \in l$, then the scalar product $c \cdot \lambda$ of the $l$-fuzzy language $\lambda$ is defined as $(c \cdot \lambda)(u)=c \wedge \lambda(u)$.

The following theorem shows that the scalar product and left, right quotients of recognizable l-fuzzy languages are recognizable.

Theorem 3.1. Let $\lambda, \lambda_{1}, \lambda_{2} \in l F\left(A^{*}\right), c \in l$. Then

(i) $c \cdot \lambda \in l F\left(A^{*}\right)$.

(ii) $\lambda_{1}^{-1} \lambda_{2}, \lambda_{2} \lambda_{1}^{-1} \in l F\left(A^{*}\right)$.

Proof. (i) Since $\lambda$ is recognizable, there exist a finite monoid $M$, an onto homomorphism $\phi: A^{*} \rightarrow$ $M$ and a $l$-fuzzy subset $\pi$ on $M$ such that $\lambda=\pi \phi^{-1}$ where $\lambda(u)=\left(\pi \phi^{-1}\right)(u)=\pi(\phi(u))$. Define a $\operatorname{map} \pi_{1}: M \rightarrow l$ by

$$
\pi_{1}(m)=c \wedge \pi(m) .
$$

Then $\pi_{1}$ is well defined. Also we have

$$
\begin{aligned}
\left(\pi_{1} \phi^{-1}\right)(u) & =\pi_{1}(\phi(u))=c \wedge \pi(\phi(u)) \\
& =c \wedge \lambda(u)=(c \cdot \lambda)(u)
\end{aligned}
$$

for all $u \in A^{*}$. Therefore $\pi_{1} \phi^{-1}=c \cdot \lambda$. Hence $c \cdot \lambda$ is recognized by $M$.

(ii) Since $\lambda_{2}$ is recognizable, there exist a finite monoid $M_{2}$, an onto homomorphism $\phi_{2}: A^{*} \rightarrow M_{2}$ and a $l$-fuzzy subset $\pi_{2}$ on $M_{2}$ such that $\lambda_{2}=\pi_{2} \phi_{2}^{-1}$ where $\lambda_{2}(u)=\left(\pi_{2} \phi_{2}^{-1}\right)(u)=\pi_{2}\left(\phi_{2}(u)\right)$. Define a map $\pi: M_{2} \rightarrow l$ by

$$
\pi(m)=\bigwedge_{v \in A^{*}}\left(\pi_{2}\left(\phi_{2}(v u)\right) \wedge \lambda_{1}(v)\right)
$$

where $m=\phi_{2}(u)$. Then we have,

$$
\begin{aligned}
\left(\pi \phi_{2}^{-1}\right)(u) & =\pi\left(\phi_{2}(u)\right) \\
& =\bigwedge_{v \in A^{*}}\left(\pi_{2}\left(\phi_{2}(v u)\right) \wedge \lambda_{1}(v)\right) \\
& \left.=\bigwedge_{v \in A^{*}}\left(\lambda_{2}(v u)\right) \wedge \lambda_{1}(v)\right)=\lambda_{1}^{-1} \lambda_{2}(u)
\end{aligned}
$$

for all $u \in A^{*}$. Hence $\lambda_{1}^{-1} \lambda_{2}=\pi \phi_{2}^{-1}$. Therefore $M_{2}$ recognizes $\lambda_{1}^{-1} \lambda_{2}$. Similarly $\lambda_{2} \lambda_{1}^{-1}$ is recognized by $M_{2}$.

Let $A$ and $B$ be finite alphabets and $\phi: A^{*} \rightarrow B^{*}$ be a homomorphism. Let $\lambda$ be a $l$-fuzzy language over $B$. The inverse of $\lambda$ under $\phi$ is a $l$-fuzzy language $\lambda \phi^{-1}$ over $A$ defined by

$$
\left(\lambda \phi^{-1}\right)(u)=\lambda(\phi(u)), u \in A^{*}
$$

The following theorem shows that the inverse homomorphic image of recognizable $l$-fuzzy language is recognizable. 
Ajitha Kumari K, Ramesh Kumar P

Theorem 3.2. Let $A, B$ be finite alphabets and let $\phi: A^{*} \rightarrow B^{*}$ be a homomorphism. If $\lambda \in$ $l F\left(B^{*}\right)$, then $\left(\lambda \phi^{-1}\right) \in l F\left(A^{*}\right)$.

Proof. Since $\lambda \in l F\left(B^{*}\right)$, there exist a monoid $M$, a homomorphism $\gamma: B^{*} \rightarrow M$ and a $l$-fuzzy subset $\pi$ of $M$ such that $\lambda=\pi \gamma^{-1}$ where $\lambda(w)=\left(\pi \gamma^{-1}\right)(w)=\pi(\gamma(w))$, where $w \in B^{*}$. Define a $\operatorname{map} \beta: A^{*} \rightarrow M$ by

$$
\beta(u)=\gamma(\phi(u)) \text {, where } u \in A^{*} .
$$

Since $\phi$ and $\gamma$ are well defined, $\beta$ is also well defined. For $u, v \in A^{*}$, we have

$$
\begin{aligned}
\beta(u v) & =\gamma(\phi(u v))=\gamma(\phi(u) \phi(v)) \\
& =\gamma(\phi(u)) \gamma(\phi(v))=\beta(u) \beta(v) .
\end{aligned}
$$

So $\beta$ is a homomorphism. Thus

$$
\begin{aligned}
\left(\pi \beta^{-1}\right)(u) & =\pi(\beta(u))=\pi(\gamma(\phi(u))) \\
& =\lambda(\phi(u))=\left(\lambda \phi^{-1}\right)(u),
\end{aligned}
$$

for all $u \in A^{*}$. So $\left(\lambda \phi^{-1}\right)=\pi \beta^{-1}$. Hence $\lambda \phi^{-1}$ is recognized by $M$.

Let $\lambda$ be a $l$-fuzzy language over $A$. The $c$-cut of $\lambda$ is the crisp language $\lambda_{c}$ defined by $\lambda_{c}=$ $\left\{u \in A^{*} \mid \lambda(u) \geq c\right\}$.

The membership function can be recovered from the level cut as $\lambda(u)=\sup \left\{c \mid u \in \lambda_{c}\right\}$. Then $\lambda=\bigvee_{c \in l}\left(c \cdot \chi_{\lambda_{c}}\right)$.

\section{Syntactic Congruence}

Let $\lambda$ be a $l$-fuzzy language over $A$. Define a relation $\left(\sim_{\lambda}\right)$ on $A^{*}$ as follows:

For $u, v \in A^{*}, u \sim_{\lambda} v$ if and only if $\lambda(p u q)=\lambda(p v q)$,

for all $p, q \in A^{*}$. Then the relation $\sim_{\lambda}$ is a congruence on $A^{*}$ called syntactic congruence of $\lambda$. The quotient monoid $A^{*} / \sim_{\lambda}=\operatorname{Syn}(\lambda)$ is called syntactic monoid of $\lambda$. We have already proved the following theorems in [1].

Theorem 4.1. For every monoid $M$ with $|M| \leq|l|$, there exist a l-fuzzy language $\lambda$ such that $M$ is the syntactic monoid of $\lambda$.

Theorem 4.2. Let $\lambda$ be a l-fuzzy language over an alphabet $A$. Then the following statements are equivalent

(i) $\lambda$ is recognizable.

(ii) $\sim_{\lambda}$ has finite index.

The following theorem gives the effect operations of $l$-fuzzy languages on the syntactic congruence.

Theorem 4.3. Let $A$ and $B$ be finite alphabets, $\lambda, \lambda_{1}, \lambda_{2}$ be l-fuzzy languages over $A, \varphi$ be a l-fuzzy language over $B, \phi: A^{*} \rightarrow B^{*}$ be a homomorphism and $c \in l$. Then the following holds.

$$
\begin{aligned}
& \text { (i) } \sim_{\bar{\lambda}}=\sim_{\lambda} . \\
& \text { (ii) } \sim_{\lambda} \subseteq \sim_{c \cdot \lambda} . \\
& \text { (iii) } \sim_{\lambda_{1} \vee \lambda_{2}}, \sim_{\lambda_{1} \wedge \lambda_{2}} \supseteq \sim_{\lambda_{1}} \cap \sim_{\lambda_{2}} .
\end{aligned}
$$


(iv) $\sim_{\lambda_{1}^{-1} \lambda_{2}}, \sim_{\lambda_{2} \lambda_{1}^{-1}} \supseteq \sim_{\lambda_{2}}$.

(v) $\phi \circ \sim_{\varphi} \circ \phi^{-1} \subseteq \sim_{\varphi \phi^{-1}}$ where $\phi \circ \sim_{\varphi} \circ \phi^{-1}$ is a congruence on $A^{*}$ defined by $(u, v) \in \phi \circ \sim_{\varphi}$ $\circ \phi^{-1} \Leftrightarrow(\phi(u), \phi(v)) \in \sim_{\varphi}$.

Proof. (i) For $u, v \in A^{*}$

$$
\begin{aligned}
(u, v) \in \sim_{\bar{\lambda}} & \Leftrightarrow \bar{\lambda}(p u q)=\bar{\lambda}(p v q), \text { for all } \mathrm{p}, \mathrm{q} \in A^{*} \\
& \Leftrightarrow \overline{\lambda(p u q)}=\overline{\lambda(p v q)} \\
& \Leftrightarrow \lambda(p u q)=\lambda(p v q) \\
& \Leftrightarrow(u, v) \in \sim_{\lambda} .
\end{aligned}
$$

Thus $\sim_{\bar{\lambda}}=\sim_{\lambda}$.

(ii)Let $u, v \in A^{*}$

$$
\begin{aligned}
(u, v) \in \sim_{\lambda} & \Leftrightarrow \lambda(p u q)=\lambda(p v q), \text { for all } \mathrm{p}, \mathrm{q} \in A^{*} \\
& \Rightarrow c \wedge \lambda(p u q)=c \wedge \lambda(p v q) \\
& \Rightarrow(u, v) \in \sim_{c \cdot \lambda} .
\end{aligned}
$$

Thus $\sim_{\lambda} \subseteq \sim_{c \cdot \lambda}$. The proof of part (iii), (iv) and (v) are similar to that given for fuzzy languages [3].

We have the following theorems.

Theorem 4.4. Let $\lambda$ be a l-fuzzy language over an alphabet $A$. Then $\sim_{\lambda}=\bigcap_{c \in l} \sim_{\lambda_{c}}$.

Proof. Let $(u, v) \in \sim_{\lambda}$, then $\lambda(p u q)=\lambda(p v q)$ for all $p, q \in A^{*}$. So for all $c \in l, \lambda(p u q) \geq c$ if and only if $\lambda(p v q) \geq c$. Thus $(u, v) \in \sim_{\lambda_{c}}$ for all $c \in l$. Hence $(u, v) \in \bigcap_{c \in l} \sim_{\lambda_{c}}$.

Conversely assume that $(u, v) \in \sim_{\lambda_{c}}$ for all $c \in l$. Then $p u q \in \lambda_{c}$ if and only if $p v q \in \lambda_{c}$ for all $p, q \in A^{*}$ and $c \in l$. That is, $\lambda(p u q) \geq c$ if and only if $\lambda(p v q) \geq c$ for all $c \in l$ and $p, q \in A^{*}$.

Thus we get $\lambda(p u q)=\lambda(p v q)$ for all $p, q \in A^{*}$. That is, $(u, v) \in \sim_{\lambda}$. Hence $\sim_{\lambda}=\bigcap_{c \in l} \sim_{\lambda_{c}}$.

Theorem 4.5. A l-fuzzy language $\lambda$ is recognizable if and only if $\operatorname{Im}(\lambda)=\left\{\lambda(u) \mid u \in A^{*}\right\}$ is a finite subset of $l$ and language $\lambda_{c}$ is recognizable for every $c \in l$.

Proof. Assume that the $l$-fuzzy language $\lambda$ is recognizable. Then by Theorem $4.2, \sim_{\lambda}$ has finite index. Also by Theorem 4.4, we have $\sim_{\lambda}=\bigcap_{c \in l} \sim_{\lambda_{c}}$. Thus for all $c \in l, \sim_{\lambda_{c}} \supseteq \sim_{\lambda}$. Hence $\sim_{\lambda_{c}}$ has finite index for all $c \in l$. So $\lambda_{c}$ is recognizable for every $c \in l$.

Since $\sim_{\lambda}$ has finite index, the congruence classes of $\sim_{\lambda}$ are finite. We may assume that these classes are $\left[u_{1}\right]_{\sim_{\lambda}},\left[u_{2}\right]_{\sim_{\lambda}}, \cdots,\left[u_{n}\right]_{\sim_{\lambda}}$. If $v, w \in\left[u_{i}\right]_{\sim_{\lambda}}$, for all $i=1,2, \cdots, n$, then $v \sim_{\lambda} w$. So $\lambda(v)=\lambda(w)$. Thus $\operatorname{Im}(\lambda)=\left\{\lambda\left(u_{1}\right), \lambda\left(u_{2}\right), \cdots, \lambda\left(u_{n}\right)\right\}$. Hence $\operatorname{Im}(\lambda)$ is finite.

Conversely assume that $\lambda_{c}$ is recognizable for all $c \in l$ and $\operatorname{Im}(\lambda)$ is finite. $\chi_{\lambda_{c}}$, the characteristic function of $\lambda_{c}$ is a $l$-fuzzy recognizable language and $\lambda=\bigvee_{c \in l}\left(c \cdot \chi_{\lambda_{c}}\right)$. Let $\operatorname{Im}(\lambda)=\left\{c_{1}, c_{2}, \cdots, c_{n}\right\}$. Then

$$
\begin{aligned}
\lambda & =\bigvee_{i=1}^{n}\left(c_{i} \cdot \chi_{\lambda_{c_{i}}}\right) \\
& =\left(c_{1} \cdot \chi_{\lambda_{c_{1}}}\right) \vee\left(c_{2} \cdot \chi_{\lambda_{c_{2}}}\right) \vee \cdots\left(c_{n} \cdot \chi_{\lambda_{c_{n}}}\right)
\end{aligned}
$$

Since $\chi_{\lambda_{c_{i}}}, i=1,2, \cdots, n$ are recognizable, $c_{i} \cdot \chi_{\lambda_{c_{i}}}$ are recognizable by Theorem 3.1. By Theorem 2.2 , their join is recognizable. Thus, $\lambda=\bigvee_{i=1}^{n}\left(c_{i} \cdot \chi_{\lambda_{c_{i}}}\right)$ is recognizable. 
Ajitha Kumari K, Ramesh Kumar P

\section{$5 \quad$ Varieties of l-Fuzzy Languages}

The notion of variety of $l$-fuzzy languages is introduced in this section.

A family of recognizable $l$-fuzzy languages is a variety of $l$-fuzzy languages, if it is closed under joins, meets, complements, scalar products, quotients, inverse homomorphic images and cuts.

Theorem 5.1. $l F\left(A^{*}\right)$, the class of all recognizable l-fuzzy languages over $A$, is a variety of l-fuzzy languages.

Proof. $l F\left(A^{*}\right)$ is closed under the Boolean operations, quotients, scalar products, inverse homomorphic images, and $c$-cut by Theorems $2.2,3.1,3.2$ and 4.5. Thus $l F\left(A^{*}\right)$ is a variety of $l$-fuzzy languages.

Theorem 5.2. The class $\left\{\chi_{L} \mid L \in \operatorname{Rec}\left(A^{*}\right)\right\}$ is a variety of l-fuzzy languages.

Proof. Take $\mathscr{A}=\left\{\chi_{L} \mid L \in \operatorname{Rec}\left(A^{*}\right)\right\}$. Let $\chi_{L_{1}}, \chi_{L_{2}} \in \mathscr{A}$, then $L_{1}, L_{2} \in \operatorname{Rec}\left(A^{*}\right)$. Since $\operatorname{Rec}\left(A^{*}\right)$ is a variety of crisp languages, $L_{1} \cap L_{2}$ and $L_{1} \cup L_{2}$ belongs to $\operatorname{Rec}\left(A^{*}\right)$. Hence $\chi_{L_{1} \cap L_{2}}$ and $\chi_{L_{1} \cup L_{2}}$ belongs to $\mathscr{A}$. Thus $\chi_{L_{1}} \wedge \chi_{L_{2}}$ and $\chi_{L_{1}} \vee \chi_{L_{2}}$ belongs to $\mathscr{A}$. Hence $\mathscr{A}$ is closed under join and meet. Let $\chi_{L} \in \mathscr{A}$ then $L \in \operatorname{Rec}\left(A^{*}\right)$. Since $\bar{L} \in \operatorname{Rec}\left(A^{*}\right), \chi_{\bar{L}} \in \mathscr{A}$. But $\chi_{\bar{L}}=\overline{\chi_{L}}$. So $\overline{\chi_{L}} \in \mathscr{A}$. Thus $\mathscr{A}$ is closed under complementation.

If $\chi_{L_{1}}$ and $\chi_{L_{2}}$ belongs to $\mathscr{A}$ then $L_{1}, L_{2} \in \operatorname{Rec}\left(A^{*}\right)$. So $L_{2} L_{1}^{-1}$ and $L_{1}^{-1} L_{2}$ are in $\operatorname{Rec}\left(A^{*}\right)$. Thus $\chi_{L_{1}^{-1} L_{2}}$ and $\chi_{L_{2} L_{1}^{-1}}$ belongs to $\mathscr{A}$. But $\chi_{L_{1}^{-1} L_{2}}=\chi_{L_{1}}^{-1} \chi_{L_{2}}$ and $\chi_{L_{2} L_{1}^{-1}}=\chi_{L_{2}} \chi_{L_{1}}^{-1}$. Hence $\mathscr{A}$ is closed under quotients.

Let $\phi: A^{*} \rightarrow B^{*}$ be a homomorphism and $L \in \operatorname{Rec}\left(B^{*}\right)$ then $L \phi^{-1}$ belongs to $\operatorname{Rec} A^{*}$. Thus $\chi_{L \phi^{-1}} \in \mathscr{A}$. But $\chi_{L \phi^{-1}}=\chi_{L} \phi^{-1}$. Hence $\mathscr{A}$ is closed under inverse homomorphic images.

Let $\chi_{L} \in \mathscr{A}$ then the $c$-cut of $\chi_{L}=\left\{u \in A^{*} \mid \chi_{L}(u) \geq c\right\}$. If $c=1$, the $c$-cut of $\chi_{L}$ is $L$. If $c=0$, then the $c$-cut of $\chi_{L}$ is $A^{*}$. For all other $c \in l$, the $c$-cut of $\chi_{L}$ is $L$. So $\mathscr{A}$ is closed under $c$-cut.

If $\chi_{L} \in \mathscr{A}$, then $c \cdot \chi_{L} \in \mathscr{A}$. Thus $\mathscr{A}$ is closed under scalar products. Hence $\mathscr{A}$ is a variety of $l$-fuzzy languages.

Since $\left\{\chi_{L}: L \in \operatorname{Rec}\left(A^{*}\right)\right\} \subset l F\left(A^{*}\right)$, the class $\left\{\chi_{L}: L \in \operatorname{Rec}\left(A^{*}\right)\right\}$ is a subvariety of $l F\left(A^{*}\right)$.

Here we define some family of recognizable languages and recognizable $l$-fuzzy languages.

Let $l F$ and $\mathscr{C}$ be varieties of $l$-fuzzy and crisp languages respectively. $l F^{c}$ be a family of recognizable languages such that for each alphabet $A$,

$$
l F^{c}\left(A^{*}\right)=\left\{L \subseteq A^{*} \mid \chi_{L} \in l F\left(A^{*}\right)\right\}
$$

and $\mathscr{C}^{f}$ be a family of recognizable $l$-fuzzy languages such that for each A,

$\mathscr{C}^{f}\left(A^{*}\right)=\left\{\lambda \in l F\left(A^{*}\right) \mid \lambda=\bigvee_{i=1}^{n} l_{i} \wedge \chi_{L_{i}}\right.$ for some $n \in \mathbb{N}, l_{i} \in l$ and $\left.L_{i} \in \mathscr{C}\left(A^{*}\right)\right\}$.

Lemma 5.3. $l F^{c}\left(A^{*}\right)$ is a variety of crisp languages.

Proof. Let $L_{1}, L_{2} \in l F^{c}\left(A^{*}\right)$ then $\chi_{L_{1}}, \chi_{L_{2}} \in l F\left(A^{*}\right)$. Since $l F\left(A^{*}\right)$ is a variety, $\chi_{L_{1}} \wedge \chi_{L_{2}}, \chi_{L_{1}} \vee$ $\chi_{L_{2}} \in l F\left(A^{*}\right)$. Thus $L_{1} \cap L_{2}, L_{1} \cup L_{2} \in l F^{c}\left(A^{*}\right)$. Hence $l F^{c}\left(A^{*}\right)$ is closed under union and intersection. Let $L \in l F^{c}\left(A^{*}\right)$ then $\chi_{L} \in l F\left(A^{*}\right)$. So $\overline{\chi_{L}}=\chi_{\bar{L}} \in l F\left(A^{*}\right)$. Thus $\bar{L} \in l F^{c}\left(A^{*}\right)$. Hence $l F^{c}\left(A^{*}\right)$ is closed under complementation.

Similarly we can prove that $l F^{c}\left(A^{*}\right)$ is closed under quotients and inverse homomorphic image. Thus $l F^{c}\left(A^{*}\right)$ is a variety of crisp languages.

Lemma 5.4. $\mathscr{C}^{f}$ is a variety of l-fuzzy languages.

Proof. For proving the family $\mathscr{C}^{f}\left(A^{*}\right)$ is closed under Boolean operations, we first proved the following identities.

(1) Let $L \subseteq A^{*}$ and $c \in l, \overline{c \wedge \chi_{L}}=\left(\bar{c} \wedge \chi_{L}\right) \vee \chi_{\bar{L}}$. 
Variety of monoid recognizable $l$-fuzzy languages

(2) For $L_{1}, L_{2} \subseteq A^{*}$, and $l_{1}, l_{2} \in l,\left(l_{1} \wedge \chi_{L_{1}}\right) \wedge\left(l_{2} \wedge \chi_{L_{2}}\right)=\left(l_{1} \wedge l_{2}\right) \wedge\left(\chi_{L_{1} \cap L_{2}}\right)$.

Let $\lambda_{1}$ and $\lambda_{2}$ belongs to $\mathscr{C}^{f}\left(A^{*}\right)$ then $\lambda_{1}=\bigvee_{i=1}^{n}\left(a_{i} \wedge \chi_{L_{i}}\right)$ for some $n \in \mathbb{N}$, $a_{i} \in l, L_{i} \in \mathscr{C}\left(A^{*}\right)$ and $\lambda_{2}=\bigvee_{i=1}^{n}\left(b_{i} \wedge \chi_{W_{i}}\right)$ for some $n \in \mathbb{N}, b_{i} \in l, W_{i} \in \mathscr{C}\left(A^{*}\right)$. Then

$$
\begin{aligned}
\lambda_{1} \wedge \lambda_{2} & =\left(\bigvee_{i=1}^{n}\left(a_{i} \wedge \chi_{L_{i}}\right)\right) \wedge\left(\bigvee_{i=1}^{n}\left(b_{i} \wedge \chi_{W_{i}}\right)\right) \\
& =\bigvee_{i=1}^{n}\left(\left(a_{i} \wedge \chi_{L_{i}}\right) \wedge\left(b_{i} \wedge \chi_{W_{i}}\right)\right) .
\end{aligned}
$$

Using the identity (2) and the fact that $\mathscr{C}\left(A^{*}\right)$ is closed under Boolean operations, we get $\lambda_{1} \wedge \lambda_{2} \in$ $\mathscr{C}^{f}\left(A^{*}\right)$.

Let $\lambda \in \mathscr{C}^{f}\left(A^{*}\right)$, then $\lambda=\bigvee_{i=1}^{n}\left(l_{i} \wedge \chi_{L_{i}}\right)$ for some $n \in \mathbb{N}, l_{i} \in l, L_{i} \in \mathscr{C}\left(A^{*}\right)$.

$\bar{\lambda}=\overline{\bigvee_{i=1}^{n}\left(l_{i} \wedge \chi_{L_{i}}\right)}=\bigwedge_{i=1}^{n} \overline{l_{i} \wedge \chi_{L_{i}}}$.

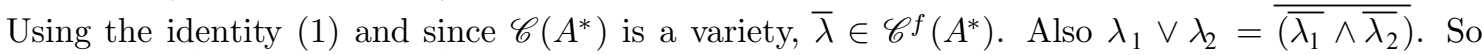
$\lambda_{1} \vee \lambda_{2} \in \mathscr{C}^{f}\left(A^{*}\right)$. Hence $\mathscr{C}^{f}\left(A^{*}\right)$ is closed under Boolean operations.

By the similar arguments given in the proof of fuzzy languages [4], $\mathscr{C}^{f}\left(A^{*}\right)$ is closed under scalar product, $c$-cut, quotients and inverse homomorphic images. So $\mathscr{C}^{f}\left(A^{*}\right)$ is a variety of $l$-fuzzy languages.

$l F^{c}$ and $\mathscr{C}^{f}$ have the following properties.

Lemma 5.5. Let $l F, l F_{1}, l F_{2}$ be varieties of l-fuzzy languages and let $\mathscr{C}, \mathscr{C}_{1}, \mathscr{C}_{2}$ be varieties of crisp languages. Then

(i) $l F_{1}\left(A^{*}\right) \subseteq l F_{2}\left(A^{*}\right)$ implies $l F_{1}^{c}\left(A^{*}\right) \subseteq l F_{2}^{c}\left(A^{*}\right)$ for every $A$.

(ii) $\mathscr{C}_{1}\left(A^{*}\right) \subseteq \mathscr{C}_{2}\left(A^{*}\right)$ implies $\mathscr{C}_{1}^{f}\left(A^{*}\right) \subseteq \mathscr{C}_{2}^{f}\left(A^{*}\right)$ for every $A$.

(iii) $l F^{c f}=l F$.

(iv) $\mathscr{C}^{f c}=\mathscr{C}$.

Proof. (i) Let $l F_{1}\left(A^{*}\right) \subseteq l F_{2}\left(A^{*}\right)$. If $L \in l F_{1}^{c}\left(A^{*}\right)$ then $\chi_{L} \in l F_{1}\left(A^{*}\right)$. So $\chi_{L} \in l F_{2}\left(A^{*}\right)$. Thus $L \in l F_{2}^{c}\left(A^{*}\right)$. Hence $l F_{1}^{c}\left(A^{*}\right) \subseteq l F_{2}^{c}\left(A^{*}\right)$.

(ii) Let $\mathscr{C}_{1}\left(A^{*}\right) \subseteq \mathscr{C}_{2}\left(A^{*}\right)$ and let $\lambda \in \mathscr{C}_{1}^{f}\left(A^{*}\right)$ then $\lambda=\bigvee_{i=1}^{n}\left(c_{i} \wedge \chi_{L_{i}}\right)$ for some $n \in \mathbb{N}, c_{i} \in$ $l, L_{i} \in \mathscr{C}_{1}\left(A^{*}\right)$. So for every $A, \lambda=\bigvee_{i=1}^{n}\left(c_{i} \wedge \chi_{L_{i}}\right)$ for some $n \in \mathbb{N}, c_{i} \in l, L_{i} \in \mathscr{C}_{2}\left(A^{*}\right)$ and thus $\lambda \in \mathscr{C}_{2}^{f}\left(A^{*}\right)$. Hence $\mathscr{C}_{1}^{f}\left(A^{*}\right) \subseteq \mathscr{C}_{2}^{f}\left(A^{*}\right)$.

(iii) $\lambda \in l F^{c f}\left(A^{*}\right)$ if and only if $\lambda=\bigvee_{i=1}^{n} c_{i} \wedge \chi_{L_{i}}$ for some $n \in \mathbb{N}, c_{i} \in l$ and $L_{i} \in l F^{c}\left(A^{*}\right)$. Since $L_{i} \in l F^{c}\left(A^{*}\right)$ for $i=1,2, \cdots, n$, we have $\chi_{L_{i}} \in l F\left(A^{*}\right)$ for $i=1,2, \cdots, n$. Then $\bigvee_{i=1}^{n} c_{i} \wedge \chi_{L_{i}} \in$ $l F\left(A^{*}\right)$ as $l F\left(A^{*}\right)$ is a variety,. That is, $\lambda \in l F\left(A^{*}\right)$. Thus $l F^{c f}=l F$.

(iv) By the definition of $\mathscr{C}^{f c}$, we have

$$
\begin{aligned}
L \in \mathscr{C}^{f c} & \Leftrightarrow \chi_{L} \in \mathscr{C}^{f}\left(A^{*}\right) \\
& \Leftrightarrow L \in \mathscr{C}\left(A^{*}\right) .
\end{aligned}
$$

Thus $\mathscr{C}^{f c}=\mathscr{C}$.

From the above results, now we obtain a one to one correspondence between the varieties of $l$-fuzzy languages and that of crisp languages.

Theorem 5.6. The mappings $l F \rightarrow l F^{c}$ and $\mathscr{C} \rightarrow \mathscr{C}^{f}$ are mutually inverse lattice isomorphisms between the lattices of all varieties of crisp languages and all varieties of l-fuzzy languages.

Proof. By Lemmas 5.3 and $5.4, l F^{c}$ is a variety of crisp languages and $\mathscr{C}^{f}$ is a variety of $l$-fuzzy languages. By Lemma 5.5, if $l F_{1} \subseteq l F_{2}$ then $l F_{1}^{c} \subseteq l F_{2}^{c}$ and if $\mathscr{C}_{1} \subseteq \mathscr{C}_{2}$ then $\mathscr{C}_{1}^{f} \subseteq \mathscr{C}_{2}^{f}$. Also, $l F^{c f}=l F$ and $\mathscr{C}^{f c}=\mathscr{C}$. Hence the theorem. 
Ajitha Kumari K, Ramesh Kumar P

\section{Variety Theorem}

In this section we obtain an Eilenberg type variety theorem for $l$-fuzzy languages.

A class $V$ of finite monoids is called a pseudovariety of monoids if it is closed under finite direct product, morphic images and subobject.

Let $\mathscr{C}$ be a variety of crisp languages, $M(L)$ be the syntactic monoid of $L$ then a variety of finite monoids $\mathscr{C}^{m}=\left\{M(L) \mid L \in \mathscr{C}\left(A^{*}\right)\right.$ for some $\left.A\right\}$ is assigned to $\mathscr{C}$. On the other hand, to a variety of finite monoid $\mathscr{M}$, a variety $\mathscr{M}^{c}=\{L$ is a recognizable language $\mid M(L) \in \mathscr{M}\}$ of recognizable language is assigned. By Eilenberg's theorem the mappings $\mathscr{C} \rightarrow \mathscr{C}^{m}$ and $\mathscr{M} \rightarrow \mathscr{M}^{c}$ are mutually inverse lattice isomorphisms between lattices of all varieties of languages and all varieties of finite monoids. By this theorem $\mathscr{C}^{m c}=\mathscr{C}$ and $\mathscr{M}^{c m}=\mathscr{M}$. Here we obtain a mutual isomorphism between varieties of $l$-fuzzy languages and varieties of finite monoids.

Let $l F$ be a variety of $l$-fuzzy languages and

$$
l F^{m}=\left\{\operatorname{Syn}(\lambda) \mid \lambda \in l F\left(A^{*}\right), \text { for some } A\right\}
$$

be a family of finite monoids.

Lemma 6.1. $l F^{m}$ is a variety of monoids.

Proof. By Theorem 4.1, lF $F^{m}$ is closed by taking submonoids and homomorphic images. Let $M_{1}$ and $M_{2}$ in $l F^{m}$, then there exist $\lambda_{1}, \lambda_{2}$ in $l F$ such that $M_{1}=\operatorname{Syn}\left(\lambda_{1}\right)$ and $M_{2}=\operatorname{Syn}\left(\lambda_{2}\right)$. By Theorem 2.2, $\lambda_{1} \vee \lambda_{2}$ and $\lambda_{1} \wedge \lambda_{2}$ are recognizable by the monoid $M_{1} \times M_{2}$. Also since $\lambda_{1}, \lambda_{2} \in l F$ and $l F$ is a variety of $l$-fuzzy languages, $\lambda_{1} \vee \lambda_{2}$ and $\lambda_{1} \wedge \lambda_{2}$ are in $l F$. Hence $M_{1} \times M_{2} \in l F^{m}$. So $l F^{m}$ is a variety of finite monoids.

Let $\mathscr{M}$ be a variety of finite monoids and for some $A$

$$
\mathscr{M}^{f}=\left\{\lambda \in l F\left(A^{*}\right) \mid \operatorname{Syn}(\lambda) \in \mathscr{M}\right\}
$$

be a family of $l$-fuzzy languages.

Lemma 6.2. $\mathscr{M}^{f}$ is a variety of l-fuzzy languages.

Proof. Let $\lambda \in \mathscr{M}^{f}$ then $\operatorname{Syn}(\lambda) \in \mathscr{M}$. By Theorem 4.3, $\sim_{\bar{\lambda}}=\sim_{\lambda}$. So $\operatorname{Syn}(\lambda)=\operatorname{Syn}(\bar{\lambda})$. Thus $\operatorname{Syn}(\bar{\lambda}) \in \mathscr{M}$. Hence $\bar{\lambda} \in \mathscr{M}^{f}$. Thus $\mathscr{M}^{f}$ is closed under complement. Similarly using the Theorems 4.4 and 4.3 , we get that $\mathscr{M}^{f}$ is a variety of $l$-fuzzy languages.

Lemma 6.3. Let lF be a variety of l-fuzzy languages, $\mathscr{C}$ be a variety of crisp languages and $\mathscr{M}$ be a variety of finite monoids. Then

(i) $l F^{c m}=l F^{m}$ and $\mathscr{C}^{f m}=\mathscr{C}^{m}$.

(ii) $\mathscr{M}^{f c}=\mathscr{M}^{c}$.

(iii) $\mathscr{M}^{c f}=\mathscr{M}^{f}$.

(iv) $l F^{m c}=l F^{c}$.

(v) $\mathscr{C}^{m f}=\mathscr{C}^{f}$.

Proof. (i) Let $\operatorname{Syn}(\lambda) \in l F^{m}$ then $\lambda \in l F\left(A^{*}\right)$. Since $l F$ is a variety of $l$-fuzzy languages, $\lambda_{c} \in$ $l F\left(A^{*}\right)$. So $\lambda_{c} \in l F^{c}$ for every $c \in l$. By Theorem 4.4, $\sim_{\lambda}=\bigcap_{c \in l} \sim_{\lambda_{c}}$. So Syn $(\lambda)$ is a subdirect product of $\operatorname{Syn}\left(\lambda_{c}\right)$ for all $c \in l$. Thus $\operatorname{Syn}(\lambda) \in l F^{c m}$. Hence $l F^{m} \subseteq l F^{c m}$. We have $l F^{c m} \subseteq l F^{m}$. Thus $l F^{m}=l F^{c m}$. By Lemma $5.5, \mathscr{C}=\mathscr{C}^{f c}$. So $\mathscr{C}^{m}=\mathscr{C}^{f c m}$. But by (i) $l F^{c m}=l F^{m}$. Thus 
$\mathscr{C}^{m}=\mathscr{C}^{f m}$

(ii) Let $L \subseteq A^{*}$, then

$$
\begin{aligned}
L \in \mathscr{M}^{f c} & \Rightarrow \chi_{L} \in \mathscr{M}^{f} \\
& \Rightarrow \operatorname{Syn}\left(\chi_{L}\right) \in \mathscr{M} \\
& \Rightarrow L \in \mathscr{M}^{c} .
\end{aligned}
$$

Thus $\mathscr{M}^{f c} \subseteq \mathscr{M}^{c}$. Also $\mathscr{M}^{c} \subseteq \mathscr{M}^{f c}$. Hence $\mathscr{M}^{f c}=\mathscr{M}^{c}$.

(iii) By Lemma 5.5, $l F^{c f}=l F$. So $\mathscr{M}^{f}=\mathscr{M}^{f c f}$. Also by (ii) $\mathscr{M}^{f c}=\mathscr{M}^{c}$. Thus $\mathscr{M}^{c f}=\mathscr{M}^{f}$.

(iv) By Lemma 5.5, $l F=l F^{c f}$. So $l F^{m c}=l F^{c f m c}$. By (i), $\mathscr{C}^{m}=\mathscr{C}^{f m}$. Thus $l F^{m c}=l F^{c m c}$. By Eilenberg's theorem $\mathscr{C}^{m c}=\mathscr{C}$. Thus $l F^{m c}=l F^{c}$.

(v) By (iii) $\mathscr{M}^{f}=\mathscr{M}^{c f}$. So $\mathscr{C}^{m f}=\mathscr{C}^{m c f}$. By using Eilenberg's theorem we get $\mathscr{C}^{m f}=\mathscr{C}^{f}$.

Now we give Eilenberg type variety theorem for $l$-fuzzy languages.

Theorem 6.4. The mappings $l F \rightarrow l F^{m}$ and $\mathscr{M} \rightarrow \mathscr{M}^{f}$ form two isomorphisms that are inverses of each other among lattices of all varieties of l-fuzzy languages and all varieties of finite monoids.

Proof. Let $l F, l F_{1}, l F_{2}$ be varieties of $l$-fuzzy languages and $\mathscr{M}, \mathscr{M}_{1}, \mathscr{M}_{2}$ be varieties of finite monoids. Let $\mathscr{M}_{1} \subseteq \mathscr{M}_{2}$ and $\lambda \in \mathscr{M}_{1}^{f}\left(A^{*}\right)$ then $\operatorname{Syn}(\lambda) \in \mathscr{M}_{1}$. So $\operatorname{Syn}(\lambda) \in \mathscr{M}_{2}$. Thus $\lambda \in \mathscr{M}_{2}^{f}\left(A^{*}\right)$. Hence $\mathscr{M}_{1}^{f}\left(A^{*}\right) \subseteq \mathscr{M}_{2}^{f}\left(A^{*}\right)$.

Also let $l F_{1}\left(A^{*}\right) \subseteq l F_{2}\left(A^{*}\right)$ for every $A$ and $\operatorname{Syn}(\lambda) \in l F_{1}^{m}\left(A^{*}\right)$. Then $\lambda \in l F_{1}\left(A^{*}\right)$. So, $\lambda \in l F_{2}\left(A^{*}\right)$. Thus $\operatorname{Syn}(\lambda) \in l F_{2}^{m}$. Hence $l F_{1}^{m}\left(A^{*}\right) \subseteq l F_{2}^{m}\left(A^{*}\right)$.

By Lemma $6.3, \mathscr{M}^{f c}=\mathscr{M}^{c}$. By Eilenberg's theorem, we have $\mathscr{M}=\mathscr{M}^{c m}$. So $\mathscr{M}=\mathscr{M}^{f c m}$. Also by Lemma $6.3, l F^{\mathrm{cm}}=l F^{m}$. Thus $\mathscr{M}=\mathscr{M}^{f m}$.

By Lemma 5.5, $l F=l F^{c f}$. By Eilenberg's theorem, $\mathscr{C}^{m c}=\mathscr{C}$. So $l F=l F^{c m c f}$. By Lemma $6.3, \mathscr{M}^{c f}=\mathscr{M}^{f}$. Hence $l F=l F^{c m f}$. Again $l F^{c m}=l F^{m}$ by Lemma 6.3. So we get $l F=l F^{m f}$. Thus $\mathscr{M}=\mathscr{M}^{f m}$ and $l F=l F^{m f}$. Hence the mappings $\mathscr{M} \rightarrow \mathscr{M}^{f}$ and $l F \rightarrow l F^{m}$ are mutually inverse lattice isomorphisms between the lattices of all varieties of $l$-fuzzy languages and all varieties of finite monoids.

\section{References}

[1] Ajitha Kumari K and Archana V P, On monoid recognizable l-fuzzy languages, International Journal of Research in Advent Technology, Vol.6, No.9, 2018, 2410-2413.

[2] G. Lallement, Semigroup and Combinatorial Applications, John-Wiley, New York, 1979.

[3] J. N. Mordeson and D. S. Malik, Fuzzy Automata and Languages; Theory and Applications, Chapman \& Hall CRC, 2002.

[4] T. Petkovic, Varieties of Fuzzy Languages, Proc. 1st Inernational Conference on Algebraic Informatics, Aristotle University of Thessaloniki, Thessaloniki, 2005.

[5] J. E. Pin, Varieties of Formal Languages, North Oxford Academic, 1986.

[6] Rakesh dube, Adesh Pandey, Retu Gupta, Discrete Structures and Automata Theory, Narosa Publishing House, New Delhi, 2007.

[7] L.A.Zadeh, Fuzzy Sets, Information and Control, No. 8, 1965, 338-353. 